\title{
Betatron phase and nonlinear lattice correction
}

\author{
Weiming Guo* \\ Lyncean Technologies Inc, Fremont, California 94538, USA \\ Yoshiteru Hidaka, Ferdinand Willeke, and Xi Yang \\ Brookhaven National Laboratory, Upton, New York 11764, USA
}

(Received 12 September 2017; revised manuscript received 10 May 2018; published 10 August 2018)

\begin{abstract}
Betatron phase measured from excited oscillation can be used to correct the beta function and the nonlinear lattice. With an improved algorithm the measurement precision has achieved $1 \mathrm{mrad}(\mathrm{rms})$, or equivalently, $0.3 \%$ of beta beat at NSLS-II. The beta beating can be corrected to $1 \%$ in less than half an hour. This precise technique can be applied to the nonlinear lattice correction as well. The betatron phase probed by an off-axis beam provides information on the sextupole strength. In comparison with the model the nominal strength can be restored. It was shown that the proposed scheme treated all the leading order resonance driving terms generated by sextupoles, as well as the detuning terms and the nonlinear chromaticity. At NSLS-II a half percent sextupole correction led to a $15 \%$ increase of the dynamic aperture.
\end{abstract}

DOI: $10.1103 /$ PhysRevAccelBeams.21.081001

\section{INTRODUCTION}

The initial magnet currents in an accelerator are calculated based on magnetic measurements; due to practical errors the settings must be fine-tuned after beam is established. Similar tuning is required routinely during operations to compensate for environmental variation or magnetic hysteresis effects. Many methods have been developed for lattice tuning. Common practice is to minimize the difference between the model prediction and the beam response to a perturbation. Two types of perturbations are commonly used. The first type is oscillatory. The beam oscillation can be excited at a fixed frequency by an $\mathrm{AC}$ dipole[1-4] or a fast corrector [5,6] or, the intrinsic betatron oscillation can be triggered by a pulsed kicker. In contrast to the oscillatory perturbation, a static trajectory deflection or orbit distortion can be induced by an orbit corrector.

In the synchrotron light source community LOCO is a widely adopted approach [7]. The method collects the orbit change in response to the variation of correctors and identifies the magnet errors by fitting all the relevant parameters such as the quadrupole and skew quadrupole strength, the beam position monitor (BPM) gain and roll, and the corrector gain and roll. LOCO has proven successful in correcting the beta function, dispersion and coupling at many facilities [8] however the final accuracy

\footnotetext{
*weiming_guo@lynceantech.com

Published by the American Physical Society under the terms of the Creative Commons Attribution 4.0 International license. Further distribution of this work must maintain attribution to the author(s) and the published article's title, journal citation, and DOI.
}

is affected by cross-talk of the errors and change of the machine condition, such as variation on the beam orbit, intensity and bunch pattern.

The beta function can be restored via betatron phase correction. The phase is obtained from the BPM turn-by-turn signal generated by an oscillating beam. This direct measurement is fast, precise, and independent of the BPM calibration. It was studied by Castro and applied at CERN in the early 1990s [9], and followed by many other facilities $[2,10-15]$. The drawback of the approach is that the phase alone cannot be used to correct dispersion and coupling. By fitting dispersion and coupling to the oscillation amplitude these lattice properties can be restored, but it is beyond the scope of the paper. Interested readers can refer to [16] for a comprehensive review of the various methods. At NSLS-II the beta-beat was corrected to several percent at commissioning using the turn-by-turn data $[17,18]$. So far the measurement precision is determined to be $\Delta \beta / \beta \approx 1 \%$ [14], and the residue being a few percent, which is significantly higher than LOCO and the other methods [8]. In this paper we will examine the algorithm and present a method to improve its accuracy.

In contrast to the success of linear lattice correction, online nonlinear characterization remains a challenge. The reason is two fold. First the multipole ( $>4$ poles) effects are small in comparison to the quadrupole focusing effect. For example, the parasitic motion induced by the sextupole driving terms is $2-3$ orders of magnitude smaller than the betatron oscillation [19]. In such a situation it is difficult to obtain precise measurement. The second obstacle is the complexity of nonlinear optimization constraints. So far most of the nonlinear tuning methods have focused on part of the nonlinear constraints, such as the resonance driving terms [20,21], the tune dependence on the amplitude [22], 
and either the beam lifetime [23] or the injection efficiency [24]. Dynamic aperture optimization requires controlling at least all the leading order terms, i.e., the leading order driving terms and the detuning terms. Partial correction of these parameters might not result in a best solution. A scheme will be proposed in the second half of the paper to treat all the leading order terms simultaneously. It is realized by correcting the focusing effect of the sextupoles while the beam orbit is offset by a corrector or by changing the rf frequency. The method has been confirmed experimentally at NSLS-II.

The theme of the paper is to correct the betatron phase and to characterize the linear and the nonlinear lattice. The paper is expanded as follows. In Sec. II the phase correction is examined by simulation and equivalence is shown to the beta function correction. It is followed by an algorithmic improvement which results in better accuracy. The demonstration of $1 \mathrm{mrad}$ of the measurement precision of the betatron phase and the application to the NSLS-II linear lattice are presented in Sec. III. The sextupole correction scheme and the experimental confirmation are described in Sec. IV. The conclusion is drawn in Sec. V. We put all the derivations in the Appendices, particularly, the error analysis of the Fourier transform, and the phase dependence on the nonlinear driving terms, as well as the proof of many statements in the main text.

\section{EXAMINATION AND IMPROVEMENT OF THE APPROACH}

\section{A. The phase correction approach}

The betatron phase advance between two BPMs indexed $i$ and $j$, is defined as

$$
\psi(i, j)=\int_{s_{i}}^{s_{j}} \frac{d s}{\beta(s)},
$$

where $\beta(s)$ is the beta function at location $s$, and $s_{i}$ and $s_{j}$ are the locations corresponding to the BPMs. For convenience a reference point can be defined, such as the injection point, then only one index is needed. In this case $\psi(i)$ means the phase advance from the reference point to the BPM $i$.

Betatron phase can be obtained from the turn-by-turn signal of the BPMs synchronized to the same turn. The motion of an electron bunch differs from a single particle due to the spread in momentum and energy [25]. A parasitic orbit oscillation can also be excited [26]. However, if the chromaticity and the oscillation amplitude are small the motion detected by BPM $i$ can be approximated as

$$
\begin{aligned}
x(n)= & g_{x x}\left[A_{x, i} \cos \left(2 \pi n \nu_{x}+\psi_{x, i}\right)+x_{c o}(n)\right] \\
& +g_{x, y}\left[A_{y, i} \cos \left(2 \pi n \nu_{y}+\psi_{y, i}\right)+y_{c o}(n)\right], \\
y(n)= & g_{y x}\left[A_{x, i} \cos \left(2 \pi n \nu_{x}+\psi_{x, i}\right)+x_{c o}(n)\right] \\
& +g_{y, y}\left[A_{y, i} \cos \left(2 \pi n \nu_{y}+\psi_{y, i}\right)+y_{c o}(n)\right],
\end{aligned}
$$

where $x(n), y(n)$ are the horizontal and vertical offsets seen by the BPM, $n$ is the turn number, $x_{c o}(n), y_{c o}(n)$ are the closed orbit offsets that can oscillate at synchrotron tune due to the kick, $\nu_{x, y}$ are the betatron tunes and $A_{x ; y, i}, \psi_{x ; y, i}$ are the initial amplitudes and phases, $g_{x x}, g_{x y}, g_{y x}, g_{y y}$ are functions of linear coupling and BPM gain and rotation.

The oscillation amplitude and phase at each BPM can be calculated by Fourier transform (FT). For example, if the averaged horizontal tune $\nu_{x}$ is obtained from the Fourier spectrum, the Fourier coefficients are calculated as

$$
\begin{aligned}
a_{i} & =\frac{2}{N} \sum_{n=1}^{N} x(n) \sin \left(2 \pi \nu_{x} n\right) \\
& =\frac{2}{N} \sum_{n=1}^{N} g_{x x} A_{x, i} \cos \left(2 \pi n \nu_{x}+\psi_{x, i}\right) \sin \left(2 \pi \nu_{x} n\right), \\
b_{i} & =\frac{2}{N} \sum_{n=1}^{N} x(n) \cos \left(2 \pi \nu_{x} n\right) \\
& =\frac{2}{N} \sum_{n=1}^{N} g_{x x} A_{x, i} \cos \left(2 \pi n \nu_{x}+\psi_{x, i}\right) \cos \left(2 \pi \nu_{x} n\right),
\end{aligned}
$$

where the other frequencies have been filtered by FT in the second step.

Applying the approximations $\sum_{n=1}^{N} \cos \left(2 \pi \nu_{x} n\right) \times$ $\sin \left(2 \pi \nu_{x} n\right) \approx 0$ and $\sum_{n=1}^{N} \cos ^{2}\left(2 \pi \nu_{x} n\right) \approx \sum_{n=1}^{N} \sin ^{2}\left(2 \pi \nu_{x} n\right) \approx$ $N / 2$, one obtains

$$
\begin{gathered}
\psi_{x, i}=\arctan \left(-a_{i} / b_{i}\right) . \\
g_{x x} A_{x, i}=\sqrt{a_{i}^{2}+b_{i}^{2}} .
\end{gathered}
$$

Where $A_{x, i}$ is the oscillation amplitude. The $g_{x x}$ factor is canceled in Eq. (4) therefore the phase $\psi_{x, i}$ does not depend on the $\mathrm{g}$ factors and BPM calibration is not necessary.

The absolute phase given by Eq. (4) depends on the starting turn. However the phase advance between BPM $i$ and $i+1$, or $\psi(i, i+1)$, depends only on the magnet settings. The phase deviation from the model is defined as [10]

$$
\phi_{i}=\psi(i, i+1)^{\mathrm{meas}}-\psi(i, i+1)^{\mathrm{mod}},
$$

where the superscript stands for "measurement" or "model." For $N$ BPMs we obtain the phase vector $\vec{\phi}=$ $\left(\phi_{1}, \phi_{2}, \ldots, \phi_{N}\right)$. In the definition $\psi(N+1)$ is needed in order to calculate $\phi_{N}$. Here $\psi(N+1)$ refers to the first BPM but the data starts from the next turn. Apparently $\vec{\phi}=\overrightarrow{0}$ corresponds to the ideal lattice. The phase error can be corrected using a response matrix calculated from the model. The turn-by-turn data can be collected within seconds hence the correction is fast. 


\section{B. Equivalence between betatron phase and amplitude}

If there are sufficient number of BPMs and the lattice is uniquely constrained, correcting $\phi$ or $\Delta \beta / \beta$ is equivalent. In order to form a local bump of $\beta$ functions, $\beta$ and $\alpha$ $\left(\alpha=-\beta^{\prime}(s) / 2\right)$ must be matched in two transverse planes at the two ends, which requires a minimum of four quadrupoles; therefore if the number of quadrupoles between two adjacent BPMs is less than four, correcting either $\phi$ or $\Delta \beta / \beta$ at the discrete BPM locations restores the lattice.

In this section we show three simulation examples to support the conclusion. At NSLS-II there are 180 BPMs and 300 quadrupoles, and the number of quadrupoles in between two adjacent BPMs is less than three. Therefore the lattice is uniquely constrained in the ideal case.

In the first example a lattice was corrected via betatron phase. The test lattice was initialized with misalignment and quadrupole strength error. The orbit was corrected. However, due to the residual magnet offset, the sextupoles contributed to the focusing error too. A response matrix of betatron phase versus individual quadrupole strength $K_{1}$ was generated, the phase error obtained from ELEGANT [27] simulation was multiplied by the inverse response matrix, and the quadrupole adjustments were obtained. Figure 1 shows the convergence of the phase and beta beat in two iterations in the horizontal plane. Similar results were obtained in the vertical plane. This example demonstrates that phase correction is as effective as beta-beat correction in a practical setup.
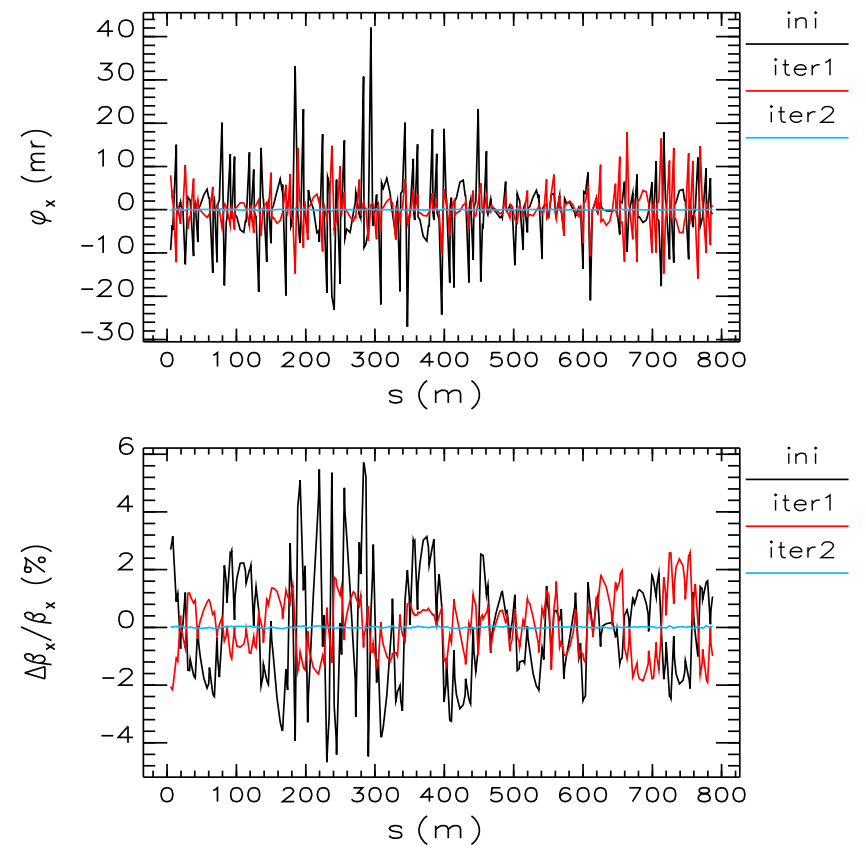

FIG. 1. Top: The horizontal phase error was corrected after two iterations for a test lattice. Bottom: The reduction of the horizontal beta beat while the phase was corrected.
The second demonstration of the equivalence is the unique conversion between the phase error and the beta beat. The beta function of an error lattice can be calculated from the measured phase advance [9]. For three consecutive BPMs 1, 2, and 3,

$$
\beta_{1}^{e} \approx \beta_{1} \frac{\cot \psi_{12}^{e}-\cot \psi_{13}^{e}}{\cot \psi_{12}-\cot \psi_{13}}
$$

where $\beta_{1}^{e}$ and $\psi_{m n}^{e}$ are the beta function and phase of the error lattice, and $\beta_{1}$ and $\psi_{m n}$ are for the unperturbed lattice. Here the superscript " $e$ " indicates the error lattice, and $\psi_{m n}$, $m, n=1,2,3$, signifies the phase advance from $m$ to $n$. The assumption of Eq. (7) is that the quadrupole error source is outside the considered region, i.e., from location 1 to 3 . Equation (7) is a good approximation even if there is local error between 1 and 3 as long as the error contribution is small compared to the total. In order to test Eq. (7) an error lattice was created with distributed quadrupole errors. The beta beat can be calculated either from the given beta function, or from the phase advance using Eq. (7). Figure 2 shows the comparison of the two approaches. The good agreement indicates the unique correspondence between the phase error and the beta beat. As proposed in [9], Eq. (7) can be used to calculate and correct the beta functions. While experimenting at NSLS-II Eq. (7) was found effective in correcting large beta beat, however, it gives unreasonable results when beta beat reaches a few percent. In simulation the agreement is always good, no matter the beta beat is large or small.

Another interesting phenomenon is that the beta beat scales linearly with the phase beat. Figure 3 shows the relation obtained from simulation. Quadrupole strength and misalignment errors were added to the NSLS-II lattice, then the standard deviations of the beta beat and phase beat were calculated while the error was increased in steps. In the plot all the BPMs were sampled for statistical calculation. The linear relations found at NSLS-II are

$$
\frac{\Delta \beta_{x}}{\beta_{x}}=2.5 \sigma_{\phi, x}, \quad \frac{\Delta \beta_{y}}{\beta_{y}}=2.8 \sigma_{\phi, y} .
$$

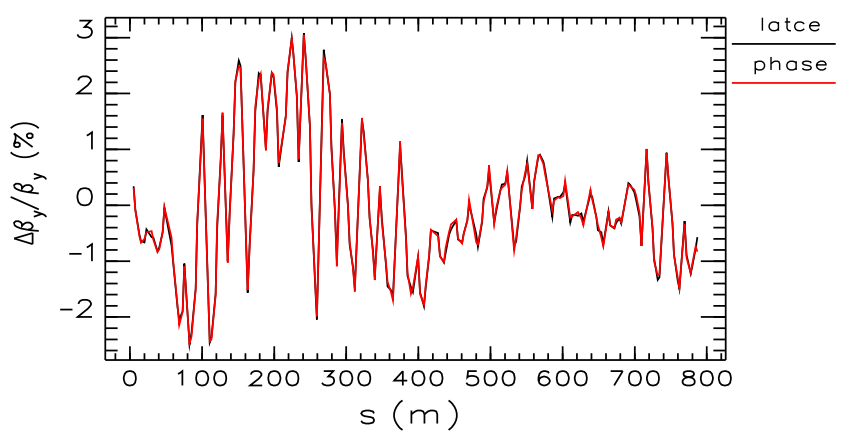

FIG. 2. The beta beat calculated from the given beta function (black) and from the phase error (red). 


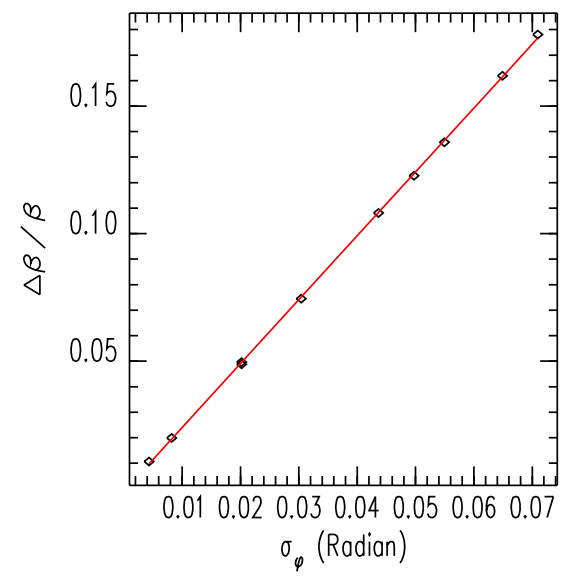

FIG. 3. The linear relation between $\sigma_{\phi}$ and $\Delta \beta / \beta$ of NSLS-II. The fitted line gives $\Delta \beta_{x} / \beta_{x}=2.5 \sigma_{\phi, x}$. All the BPMs were sampled for statistics.

The ratio was different if the sampling included other elements even though the relation remained linear. But this is not a problem because during experiment the sampling is always taken only at the BPMs. A similar linear relationship was reported at another facility [10].

\section{Improved Fourier transform}

In this section the improvement of the phase analysis algorithm will be discussed. The uncertainty of Eq. (4) can be calculated as

$$
\begin{aligned}
\Delta \psi & =\sqrt{\left(\frac{\partial \psi}{\partial a} \Delta a\right)^{2}+\left(\frac{\partial \psi}{\partial b} \Delta b\right)^{2}} \\
& =\frac{a / b}{1+(a / b)^{2}} \sqrt{(\Delta a / a)^{2}+(\Delta b / b)^{2}} .
\end{aligned}
$$

The uncertainty due to the signal noise is given by,

$$
\left\langle\Delta a^{2}\right\rangle=\frac{4}{N^{2}} \sum_{n=1}^{N}\left\langle\Delta x^{2}\right\rangle\left\langle\sin ^{2} \nu_{x} n\right\rangle=\frac{2}{N}\left\langle\Delta x^{2}\right\rangle,
$$

However $a^{2} \approx\left\langle x^{2}\right\rangle$, therefore $\left(\frac{\Delta a}{a}\right)^{2} \approx \frac{2}{N} \frac{\left\langle\Delta x^{2}\right\rangle}{\left\langle x^{2}\right\rangle}$. The same is true for $(\Delta b / b)^{2}$.

The fitting frequency error contributes to the calculation of the amplitude and phase and is analyzed in Appendix A. The contributions are $\Delta a / a=\Delta b / b \approx f(\nu, \Delta \nu, \psi)$ and $\Delta \psi=(N+1) \pi|\Delta \nu|$. Here we use $\nu$ to represent $\nu_{x}$ or $\nu_{y}$. Derivation of these two terms can be found in Appendix A. The $(N+1) \pi|\Delta \nu|$ term can reach $\pi / 2$ in a Fourier transform since the intrinsic frequency error is $1 /(2 N)$. This error is the same for all the BPMs and it is canceled in Eq. (6). $f(\nu, \Delta \nu, \psi)$ induces a phase error of several milliradians and is not canceled. In summary, the total error is given by

$$
\begin{aligned}
\Delta \psi= & \frac{1}{1+(a / b)^{2}} \sqrt{\frac{4}{N} \frac{a^{2}}{b^{2}} \frac{\sigma_{x}^{2}}{x^{2}}+2 f^{2}(\nu, \Delta \nu, \psi)} \\
& +(N+1) \pi|\Delta \nu|,
\end{aligned}
$$

where $\sigma_{x} / \bar{x}$ is the BPM noise to signal ratio. The last term is systematic error therefore is presented separately. The error contribution from the other sources will be discussed later.

The algorithm is to be improved in two steps. The first step is to finely analyze the tune so the $\Delta \nu$ related terms can be minimized in Eq. (11). We found NAFF [28] is the most accurate method for this purpose. The idea is to search for the frequency of the maximum amplitude that fits the oscillation data. A Hann window significantly improves the precision. With NAFF $\Delta \nu$ is reduced to $1 / N^{2}$ or even $1 / N^{3}[28]$.

Once the tune is determined, the phase can be obtained by a similar search, i.e., scanning the phase $\psi$ to minimize the function $\sum_{n}^{N} x_{n} \sin (2 \pi \nu n+\psi)$. The phase correction can also be found analytically. Assume the signal can be fitted by the following function

$$
f_{n}=A e^{-\alpha_{T} \theta_{n}} \cos \left(\nu \theta_{n}+\psi+\epsilon\right),
$$

where $\theta_{n}=2 \pi n, A$ and $\alpha_{T}$ are the amplitude and the damping coefficient, which are pre-determined from fitting the oscillation amplitude. $\epsilon$ is the correction to the phase obtained from Eq. (4). A least square fit to the signal $x_{n}$ gives

$$
\epsilon=\frac{e_{1}-e_{2}}{e_{3}-e_{4}}
$$

where

$$
\begin{aligned}
& e_{1}=\sum_{n} \frac{A}{2} \exp \left(2 \alpha_{T} \theta_{n}\right) \sin 2\left(\nu \theta_{n}+\psi\right), \\
& e_{2}=\sum_{n} x_{n} \exp \left(\alpha_{T} \theta_{n}\right) \sin \left(\nu \theta_{n}+\psi\right), \\
& e_{3}=\sum_{n} x_{n} \exp \left(\alpha_{T} \theta_{n}\right) \cos \left(\nu \theta_{n}+\psi\right), \\
& e_{4}=\sum_{n} A \exp \left(2 \alpha_{T} \theta_{n}\right) \cos 2\left(\nu \theta_{n}+\psi\right) .
\end{aligned}
$$

The result from Eq. (13) is very close to the numerical search if the signal is regular. $\epsilon$ is not sensitive to the precision of $A$ and $\alpha_{T}$ hence iteration of fitting is not necessary. The amplitude of $\epsilon$ is on the order of 1 milliradian. Therefore the correction is critical for our purpose.

A simple simulation was carried out to test the algorithm. In this case a noise signal of the amplitude $A \cdot R(n)$ is added to Eq. (12). Here $R(n)$ is random noise uniformly distributed in $(-|R|,|R|)$. The amplitude, frequency and phase 


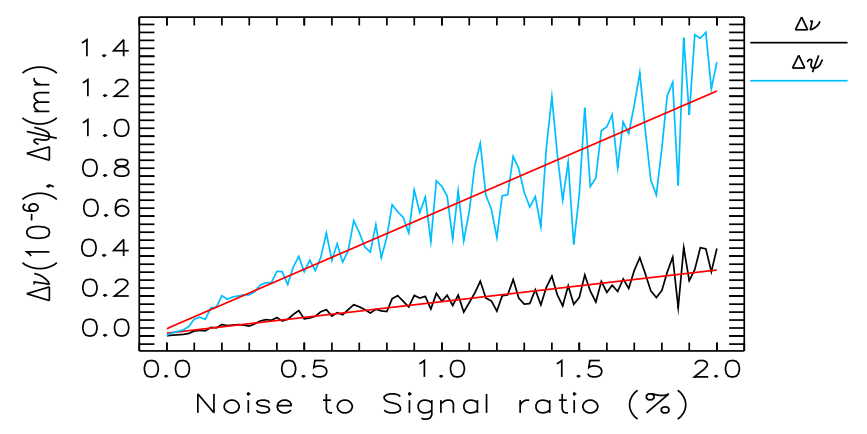

FIG. 4. The tune error $(\Delta \nu)$ and phase error $(\Delta \psi, \operatorname{mrad})$ grow linearly with noise-to-signal ratio $(\mathrm{R}, \%)$. Here $\nu$ and $\psi$ are obtained from fitting of 1000 points. 20 seeds were averaged for each $\mathrm{R}$ value. The fitted lines are given by $\Delta \nu=1.06 \times 10^{-5} \cdot R$, and $\Delta \psi=3.58 \times 10^{-2} \cdot \mathrm{R}$ (Radian). Therefore $\Delta \psi=\pi(N-1) \Delta \nu+$ $0.08 \frac{2 R}{\sqrt{N}}$, in reasonable agreement with Eq. (11), which gives $\Delta \psi=\pi(N-1) \Delta \nu+0.12 \frac{2 R}{\sqrt{N}}$. Here $a / b=3.9$ and $f(\nu, \Delta \nu, \psi)=0$ were used.

were analyzed and compared with the input values. Figure 4 shows the difference versus the noise ratio $R$.

At NSLS-II the BPM turn-by-turn noise for a $10 \mathrm{~mA}$ 100-bunch train is about $10 \mu \mathrm{m}$ [29], or $1 \%$ if the oscillation amplitude is $1 \mathrm{~mm}$. This corresponds to $\Delta \nu \sim$ $10^{-7}$ in Fig. 4. The order of magnitude agrees with the frequency deviation found from the statistics of 180 BPM signals collected during one excitation event. The phase correction given by Eq. (13) is about $0.5 \mathrm{mrad}$ in this case.

\section{LINEAR LATTICE CORRECTION}

\section{A. Accuracy and application to NSLS-II}

The phase correction method was applied on NSLS-II. First we studied the measurement accuracy. The phase vector was measured while the strength of a quadrupole (QH1G2C02A) was varied in steps. Figure 5 shows the comparison of the measured and calculated phase vector for a magnet current change of $0.5 \mathrm{~A}$. The agreement is best achieved if the measured value is multiplied by a fitting factor of $f_{x}=1.015$ to account for the beta function deviation at the quadrupole, hysteresis, transfer-function uncertainty, and inaccuracy of the hard-edge model. The error bar of $1.7 \mathrm{mrad}$ was determined from the standard deviation of 10 repetitive measurements; however, the difference from the model is calculated to be $\sigma_{\phi}=$ $0.7 \mathrm{mrad}$ (rms of $180 \mathrm{BPMs}$ ). The greater measurement fluctuation is probably caused by orbit drifting, power supply ripple, and rf jitter but averaging clearly reduces the effects of these errors. The BPM gain and roll error are on the order of $10^{-2}$ and were not corrected during the analysis. The $\sigma_{\phi} \sim 1 \mathrm{mrad} \mathrm{rms}$ accuracy $\left(\sim 10^{-3}\right)$ indicates the measurement is not affected by the BPM gain and roll error.
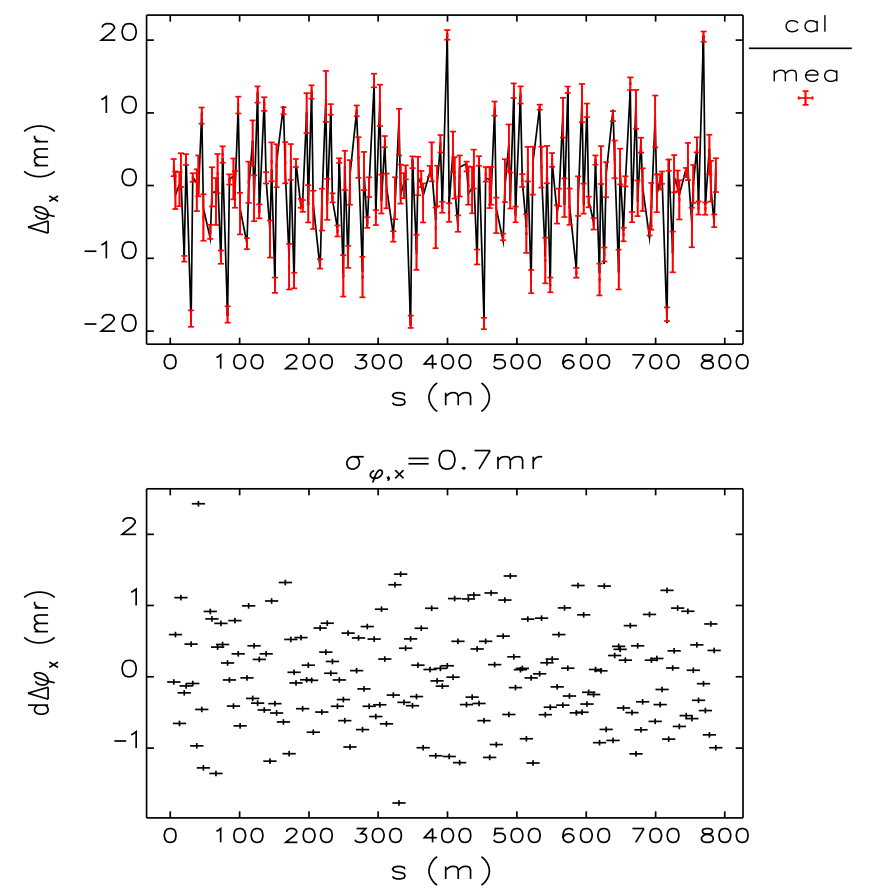

FIG. 5. Top: Comparison of the horizontal phase change obtained from measurement and calculation when the current of QH1G2C02A is changed by $0.5 \mathrm{~A}$, or, $\Delta K_{1}=8.4 \times 10^{-4} \mathrm{~m}^{-2}$. Bottom: The phase difference between measurement and calculation.

The same measurement was repeated at $\Delta I=0.25 \mathrm{~A}, 1$ A, 2 A, and the deviation from the model is shown in Fig. 6 for both transverse planes. $\sigma_{\phi}$ ranges from 0.3 to $1.2 \mathrm{mrad}$, with vertical plane being slightly smaller. The same data was analyzed by two methods: the standard Fourier transform with tune read from Fourier spectrum, and the improved algorithm, i.e., fit for the tune and the phase. The improvement of accuracy is apparent in both planes. We would like to point out that the phase deviation is partly caused by the residual beta beat in the lattice [see Eq. (B2)]. In this case the error increases with $\Delta I$, which is seen on the last two points of Fig. 6. From Eq. (8), 1 mrad phase error
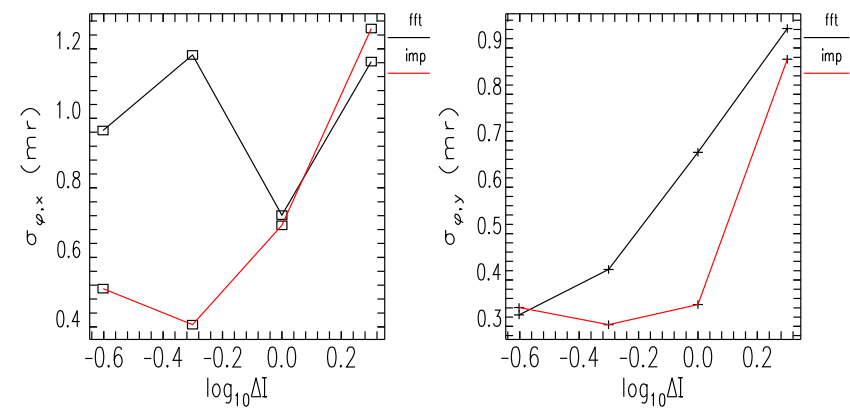

FIG. 6. The standard deviation of the phase discrepancy when the quadrupole current is changed by $\Delta I=0.25,0.5,1,2$ A. Black: analyzed with standard FT, red: with the improved algorithm. 

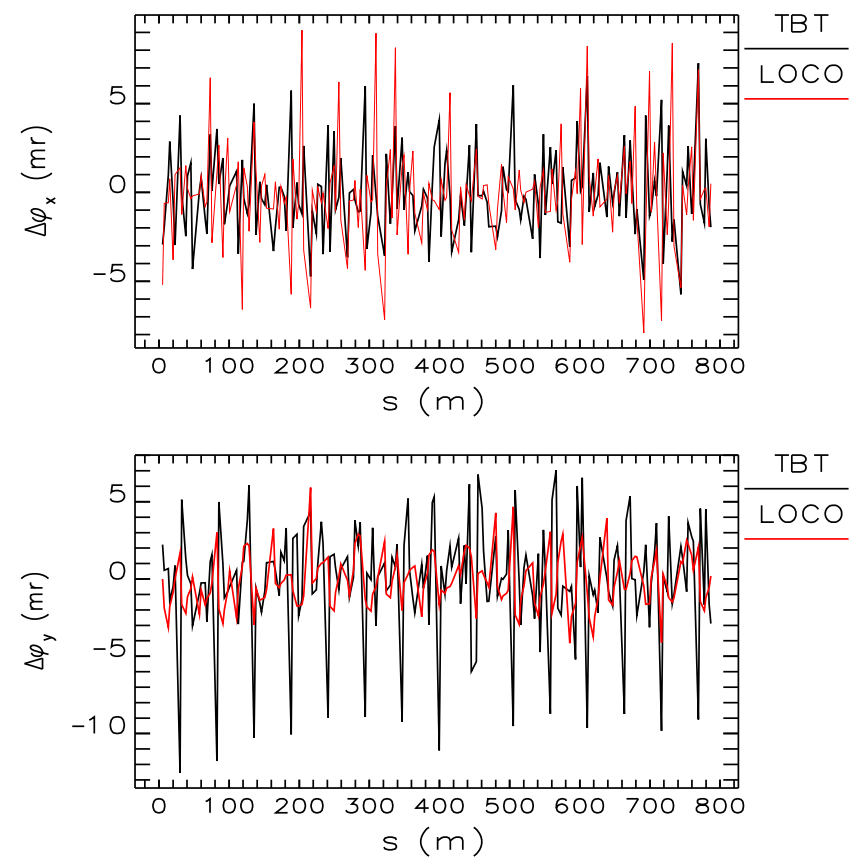

FIG. 7. Typical residual phase error measured from the turn-byturn signal and by LOCO.

corresponds to about $0.3 \%$ of beta beat; therefore the accuracy is satisfactory for NSLS-II when iteration is applied.

The phase error of the lattice can be corrected using the model response matrix. At NSLS-II the residual peak value reduces to below $10 \mathrm{mrad}$ after a few iterations [30]. The process takes about $20-30 \mathrm{~min}$ due mostly to repetitive measurements. The corrected lattice was once measured by LOCO [7] and the residual beta beat was determined to be about $0.9 \%$ (x) and $0.6 \%(\mathrm{y}) \mathrm{rms}$. The residues are similar if the formulas in Eq. (8) are applied. LOCO provides a fitted lattice so the phase error can be calculated. The residual phase error measured from the turn-by-turn signal and by LOCO are compared in Fig. 7. The order of magnitude agrees in the horizontal plane however not in the vertical plane. The negative peaks in the vertical plane occurring at the peak dispersion locations are not yet understood.

It is interesting to mention that the phase analysis method has been successfully applied at ESRF [15] with a reference to our conference paper. Similar results have been obtained. The measurement precision, or the uncertainty among measurements, was determined to be $\Delta \beta / \beta=0.2-0.4 \%$, which is close to the $0.3 \%$ obtained at NSLS-II. The discrepancy of beta-beating is on the order of $1 \%$ between the phase method and the orbit based method, which is similar to our results.

Phase measurement can be used to eliminate aliasing. Aliasing is a common problem in spectrum analysis. When the Fourier transform is applied to a signal

$$
x(\theta)=A \cos (\nu \theta+\phi),
$$

the amplitude is identical for the tune $\nu$ or $1-\nu$. Therefore the spectrum itself cannot distinguish the two cases. However, if the phase is known, the situation is clarified. One can verify that for the signal in Eq. (14), the phase is $\phi$ or $-\phi$ if the fitting tune is $\nu$ or $1-\nu$, respectively.

In an accelerator the tune can be determined if the turnby-turn data is collected at two adjacent BPMs. The approximate phase advance between the two BPMs can be obtained from the model. If the tune is correct (i.e., $\nu$ ) the phase advance should agree, otherwise (i.e., $1-\nu$ ) the calculated phase advance has an opposite sign.

\section{B. Discussion of the errors}

The betatron phase is affected by the sextupoles and skew quadrupoles even if the beam orbit is on-axis. In order to account for the sextupole contribution in the model, tracking must be used for the model phase calculation. The oscillation amplitude must be the same as that applied in the experiment. The skew quadrupole contribution is difficult to model because the error sources are unknown. In order to minimize the error during the experiment, the linear coupling was corrected; and the probing oscillation was excited only in one plane at a time.

Many factors affect the analysis. The main error source comes from the deformation of the sinusoidal oscillation, which can be induced by decoherence, damping, nonlinearity, chromatic modulation, and interference from other frequencies. The fitting loses precision when the amplitude quickly damps or if it is heavily modulated. At NSLS-II decoherence starts at very low single bunch current. During the experiment a low beam current of $2 \mathrm{~mA}$ was stored in a 100 bunch-train to avoid collective effects. In order to mitigate nonlinear decoherence, 1 and $0.5 \mathrm{~mm}$ kick amplitudes were used for the horizontal and the vertical plane, respectively. These amplitudes were picked to balance the noise ratio and the nonlinear deformation.

The precision is also affected by the companion oscillations. For example, if synchrotron side bands are present the betatron frequency calculated by NAFF shifts slightly. This is artificial and can be verified in simulation. The error becomes negligible if the frequencies are far apart. We found that at peak dispersion location the phase error is larger compared to the other locations if there is coherent synchrotron oscillation.

BPM timing and alignment introduce error as well. The BPMs must be synchronized to the same turn. Otherwise it causes an error of $2 n \nu \pi$, where $n$ is the number of lapsed turns, and $\nu$ is the tune. The beam arrival time jitter does not affect the measurement because the signal is collected and analyzed for the full turn by the electronics. The contribution from BPM longitudinal misalignment is small. For example, if the longitudinal shift is $\Delta l=0.5 \mathrm{~mm}$ and the beta function $\beta_{p}=10 \mathrm{~m}$, the phase error is $\Delta l / \beta_{p}=0.05 \mathrm{mrad}$, which is negligible. 
So far the correction has been limited to the beta functions. The method can be extended to include dispersion. In that case the amplitude must be included as well as the phase. In principle if the beta functions are corrected dispersion should depend on the dipole strength only. This idea will be explored in the future. Coupling cannot be corrected based on the phase measurement. Because of these reasons, phase correction is not a stand-alone linear lattice correction method. However, the method is a good complement to the other methods, for example, in case a quick beta function correction is needed.

\section{NONLINEAR LATTICE CORRECTION}

\section{A. Nonlinear correction method and the target functions}

Phase correction can be used to restore the nonlinear lattice as well. The idea is to measure and correct the focusing effect of the sextupoles while orbit is offset from the magnet center. There are two ways to offset the beam in the sextupoles. One way is to create an orbit wave by altering a horizontal orbit corrector. However some of the sextupoles might have close-to-zero offset; hence repetition must be done at a minimum of two correctors that are separated by $\pi / 2$ phase advance. Similar to the LOCO approach, adding more correctors improves precision and minimizes degeneracy. The second way is to run the beam off-momentum. In Appendix B it is shown that in the first case phase advances are functions of the geometric driving terms, and in the second case they depend on the chromatic driving terms. Therefore both cases are needed for a complete nonlinear characterization. The peak orbit deviation must be large enough to excite measurable focusing effects. The bending angle or the momentum offset can be determined from fitting the orbit change at all the BPMs. A study lattice can be created with the applied bending angle or the momentum offset.

The phase vector must be measured for the perturbed and unperturbed lattices. The phase change induced by the sextupoles are given by

$$
\Delta \psi_{i}=\psi(i, i+1)^{\text {perturbed }}-\psi(i, i+1)^{\text {unperturbed }}
$$

The difference between calculation and measurement is

$$
\phi_{i}=\Delta \psi_{i}^{\text {measurement }}-\Delta \psi_{i}^{\text {calculation }}
$$

$\phi_{i}$ is the error to be corrected.

Dynamic aperture optimization requires the necessary nonlinear constraints be: the leading order driving terms, the amplitude dependent tune shift terms, and the first, second and third order chromaticity [31]. As discussed in Appendix B, the leading order driving terms are constrained by the phase correction. For completeness the following terms are included into the penalty vector:

$$
\vec{F}=\left(\phi_{1}, \phi_{2}, \ldots, \phi_{N}, \frac{d \nu_{x}}{d J_{x}}, \frac{d \nu_{x}}{d J_{y}}, \frac{d \nu_{y}}{d J_{y}}, \xi_{x}^{(1)}, \xi_{y}^{(1)}, \xi_{x}^{(2)}, \xi_{y}^{(2)}, \xi_{x}^{(3)}, \xi_{y}^{(3)}\right)^{T}
$$

The response matrix with elements $m_{m, n}=\frac{\partial F_{m}}{\partial K_{2 n}}$ are calculated numerically. The phase $\left(\phi_{i}\right)$ related terms of the response matrix is calculated from the study lattice with orbit offset. The response matrix for the detuning terms and the chromatic terms should be obtained from the original lattice. This routine should be applied to all the study lattices while switching correctors or changing momentum. The response matrices and the phase error vectors must be assembled together. Similar to the linear correction, the error phase vector is multiplied by the inverse response matrix and the correction is obtained.

We would like to emphasize that this method restores the sextupole strength to the model value which differs from online optimization methods such as the lifetime [23] or injection efficiency [24] optimization. The objective of these methods is to improve one aspect of the accelerator performance, and the final lattice might deviate from the model.

\section{B. Demonstration at NSLS-II}

In the following section NSLS-II is used again to illustrate the method. The first step is to create orbit distortion. The phase vector must be measured with or without the distortion so the difference caused by the sextupoles can be obtained. Figure 8 shows the two types of orbit changes and the fitting results. The fitted angle or the momentum offset are used to generate study lattices.

Once the study lattice is generated the phase difference between the study lattice and the original lattice can be calculated which gives the model value of the sextupoleinduced phase change. The phase error can be obtained from comparison with measurement. Figure 9 shows the measured phase change caused by the orbit offsets from Fig. 8 and the model prediction.

The same measurement was repeated in 24 cases, particularly, with 20 correctors and at 4 momentum-offsets. Sextupole response matrices were calculated for all the perturbed lattices. The assembled response matrix was used to compute the sextupole correction. The calculated correction is shown in Fig. 10. The repeating pattern is due to the sextupoles being powered in series in every 6 cells. The nonzero average of $0.1 \mathrm{~m}^{-3}$, or, $-0.27 \%$, is probably 

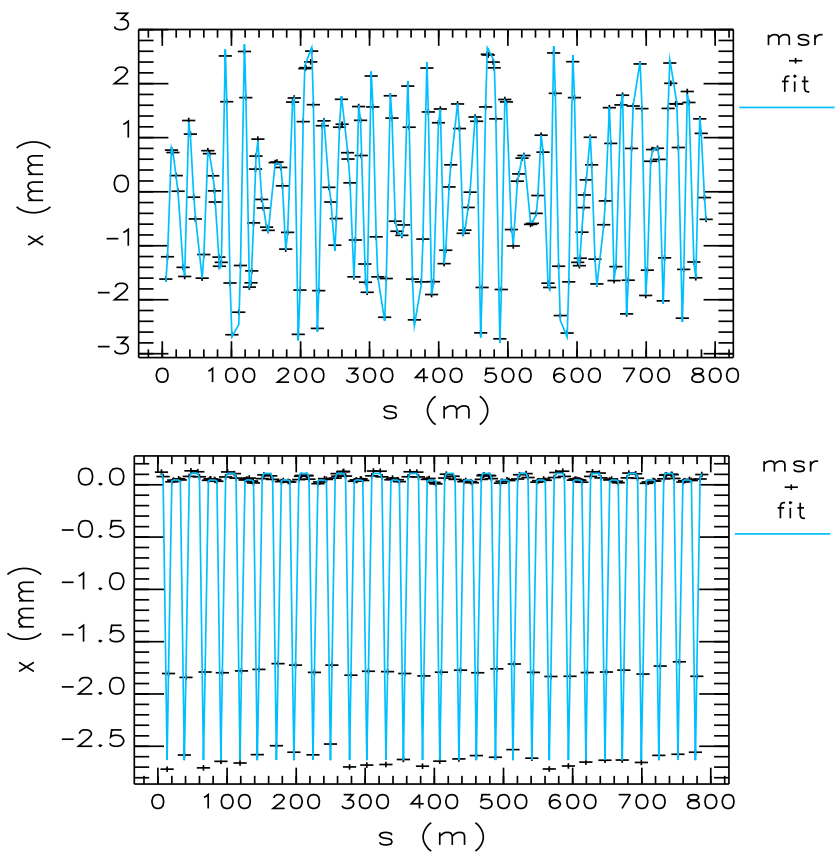

FIG. 8. The measured and the fitted orbit. Top: The fitted bending angle is $0.18 \mathrm{mrad}$. Bottom: The fitted momentum offset is $-0.6 \%$.
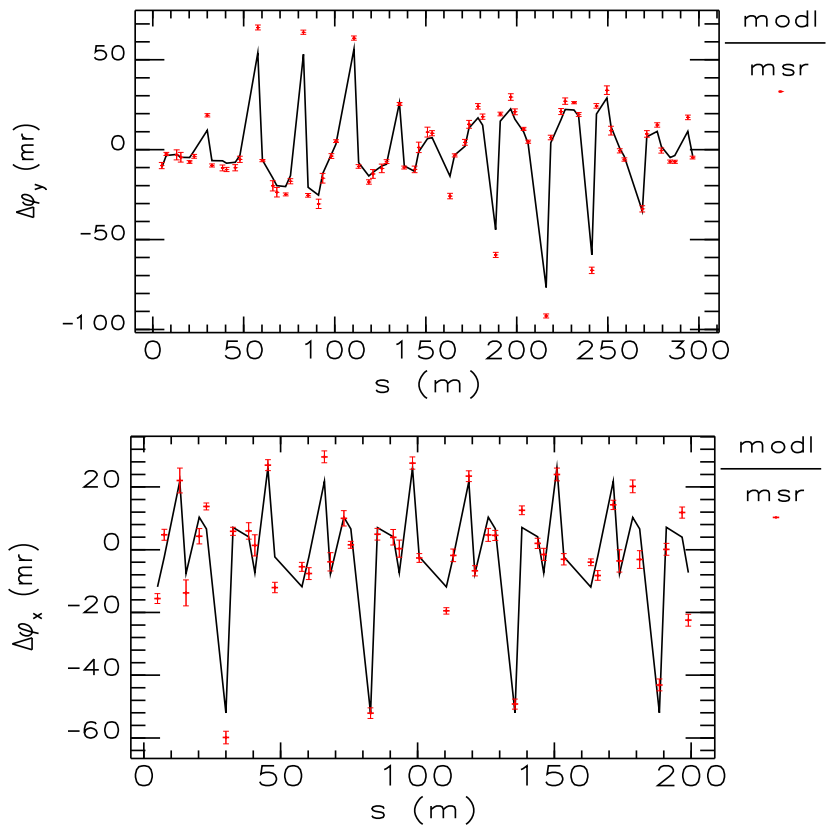

FIG. 9. The measured and the calculated phase change caused by the sextupoles. Top: The corrector was changed by $0.18 \mathrm{mrad}$. Bottom: The momentum offset was $-0.6 \%$. The error bar was obtained from 6 measurements. Only one plane in part of the ring is shown for better view.

caused by BPM or sextupole calibration error. The BPM global gain error leads to an error in the bending angle and must be corrected. The BPM gain correction was applied in our analysis.

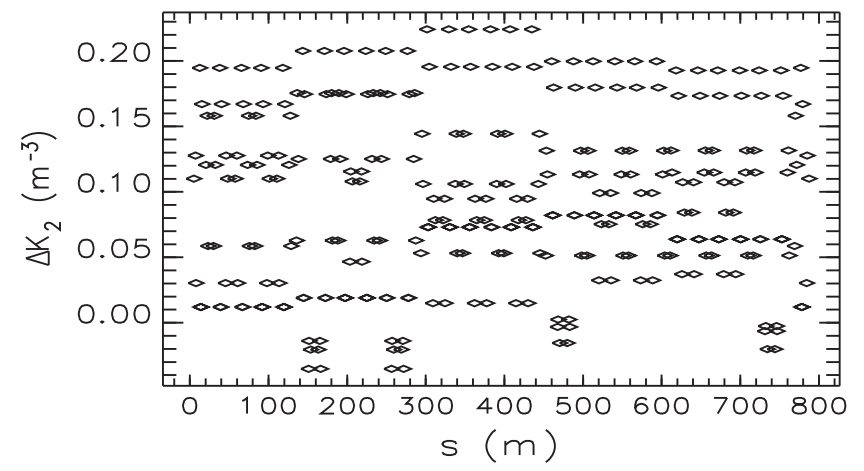

FIG. 10. The calculated error of the sextupoles powered in 54 circuits. With several exceptions, each circuit connects 6 sextupoles; therefore the neighboring 6 points are of the same value.

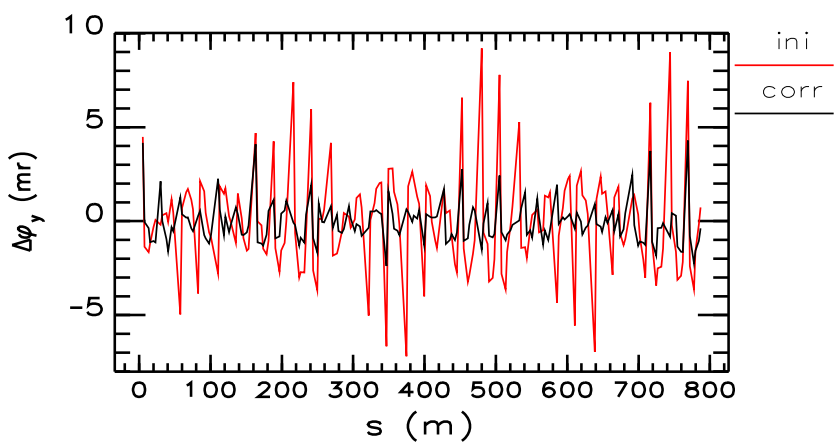

FIG. 11. The initial and the residual phase error after one round of correction. Only one plane is shown.

The correction of the phase error converged quickly during the experiment. Figure 11 shows the error before and after one iteration for one of the study lattices.

The rms phase errors before and after correction for the study lattices are plotted in Fig. 12. Here the $s$ axis corresponds to the location of each horizontal corrector that is used to generate orbit distortion. The residual error shown in the plot is about $2.5 \mathrm{mrad}$. The horizontal residual has similar amplitude. The off-momentum phase error reduction is small, due probably to the large dispersion deviation from the nominal value (see Fig. 8). It is worth mentioning that the residual phase shows dependence on $\sqrt{\beta_{x}}$. This is because the same bending angle was applied for all the correctors; however the beta functions differ at the individual corrector families, which results in orbit distortion proportional to $\sqrt{\beta_{x}}$. The phase deviation depends on the orbit offset in the sextupole which explains the pattern in Fig. 12.

The correction of the detuning terms was only partly successful. Table I shows the comparison of the results. The $d \nu_{x} / d J_{x}$ and $d \nu_{y} / d J_{y}$ terms were corrected very well, however, there was almost no change to the 2 nd order chromaticity, and there was a blow-up on the 3 rd order. The correction of the nonlinear chromaticity probably needs more iterations. 


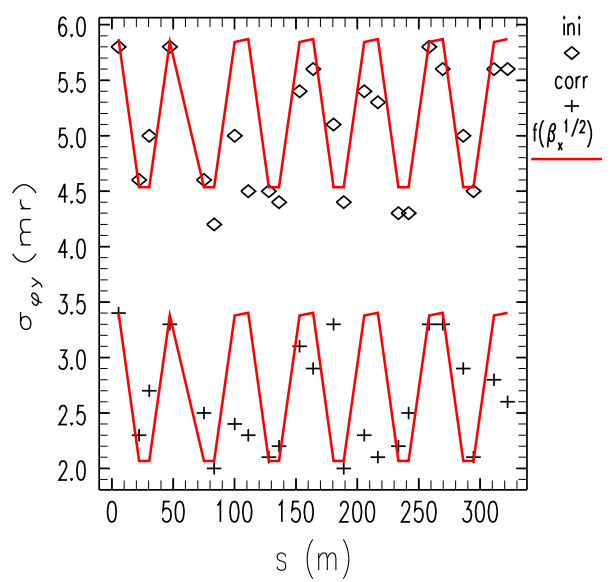

FIG. 12. The vertical phase error before and after one correction. Each point corresponds to a study lattice which is perturbed by one corrector at that location. The diamond and the plus sign signify the initial and the residual error, respectively. The red lines are fittings to the $\sqrt{\beta_{x}}$. The bending angle is about $0.2 \mathrm{mrad}$ for all the correctors.

The dynamic aperture (DA) was measured before and after sextupole correction. The method to determine DA is as follows. First a 200-bunch train (15\% of full fill) of $20 \mathrm{~mA}$ current was stored, and the beam was kicked by a one-turn half-sine-wave pulsed magnet (pinger). The pinger timing was adjusted so the maximum is aligned to the bunch train center to ensure a uniform kickamplitude to all the bunches. Then the beam was kicked by the pinger with strength increased in steps. The stored current was monitored by a high-precision DC current transformer (DCCT) and the readings were used to calculate the loss rate. The induced oscillation was recorded by the BPMs and the amplitude was used to determine DA. In principle a 2D dynamic aperture mapping can be obtained with the horizontal and the vertical pingers. Due to time limitation only the horizontal axis was scanned. The results are compared in Fig. 13. The on-axis dynamic aperture is clearly enlarged. As a result the injection efficiency was improved. However we did not observe significant lifetime change.

We tested the sextupole correction algorithm with a known error. An error lattice was created by lowering one sextupole power supply by $10 \mathrm{~A}(\approx 20 \%)$. The corresponding sextupole strength change was $\Delta K_{2}=4.2 \mathrm{~m}^{-3}$. Then the phase vector and the nonlinear detuning terms were measured and compared to the original lattice.

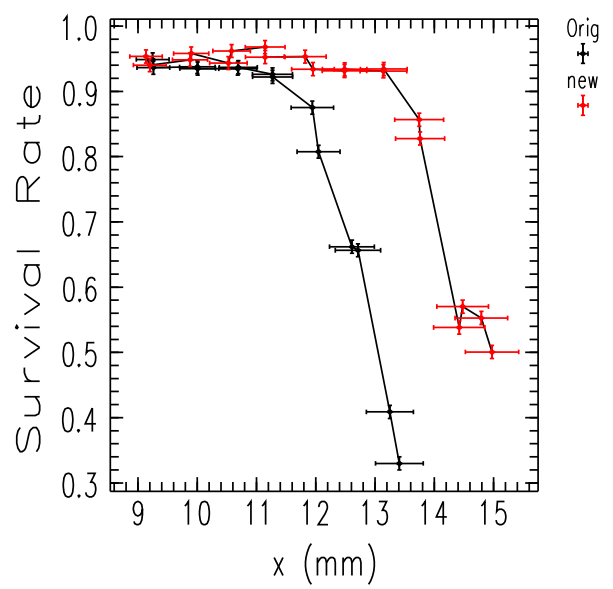

FIG. 13. Loss occurs when the stored beam is kicked by the pinger. The survival rate $\left(I_{n} / I_{n-1}\right.$, with $n$ as the step number $)$ is shown versus the oscillation amplitude measured by the BPMs. The error bar is due to a few percent of the calibration error in the BPM and the DCCT. The improvement of the on-axis DA is about $2 \mathrm{~mm}$, or $15 \%$.

The algorithm was used to identify the error, and the results are plotted in Fig. 14. Among 54 power supplies the algorithm clearly points to the changed one. The amplitude also agrees very well with the input value. The background error of $\Delta K_{2}=0.5 \mathrm{~m}^{-3}$ is due to the inversion algorithm. It can be lowered if a smaller number of eigenvalues are kept however the amplitude at index 39 will also be smaller. The actual accuracy is $\Delta K_{2}<0.1 \mathrm{~m}^{-3}$, as shown in Fig. 10. This is a common problem of the inverse matrix calculation. Iteration must be performed to reduce the noise level.

\section{Physical meaning of the correction and error minimization}

Even though it was claimed in the previous section that correction of the phase of the perturbed lattice is to restore the nonlinear driving terms, the approach can also be understood as beta function or phase correction for the offmomentum lattice or for the orbit-perturbed lattice. The significance of the second case lies in the fact that the closed orbit distorted by a single corrector is essentially a betatron oscillation wave. Therefore the correction restores the transfer matrix for the oscillating beam. In this case the transfer matrix, which depends on the corrected beta function and phase, differs from the linear transfer matrix

TABLE I. Comparison of the detuning terms.

\begin{tabular}{lccccccr}
\hline \hline & $d \nu_{x} / d J_{x}$ & $d \nu_{y} / d J_{y}$ & $d \nu_{y} / d J_{x}$ & $d \nu_{x}^{2} / d p^{2}$ & $d \nu_{y}^{2} / d p^{2}$ & $d \nu_{x}^{3} / d p^{3}$ & $d \nu_{y}^{3} / d p^{3}$ \\
\hline Initial & -1624 & -16831 & 1337 & -126 & 22 & 489 & -92 \\
Corrected & -4601 & -8055 & -890 & -126 & 21 & 3116 & -850 \\
Nominal & -4944 & -9710 & -1428 & -157 & 28 & 1405 & -76 \\
\hline \hline
\end{tabular}




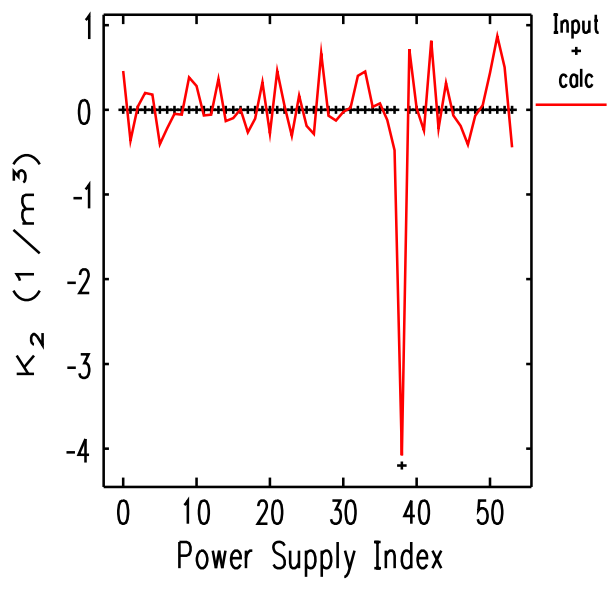

FIG. 14. The cross shows the changed sextupole power supply (index 39, SL1C21) with $\Delta K_{2}=4.2 \mathrm{~m}^{-3}$, and the red curve is the sextupole correction identified by the algorithm.

in that it includes the focusing effect of the sextupoles. Apparently the method is capable of correcting the feeddown focusing effect of any higher-order multipoles.

The accuracy of the correction depends on many factors. First the method corrects the focusing strength which is proportional to $\beta_{x, y} K_{2} \Delta x_{c . o}$. Therefore errors of $\beta$ function and closed orbit propagate to $K_{2}$. For this reason it is important to correct the $\beta$ function to high precision. Treating all sextupoles in one family as one variable provides better precision because the $\beta$ error is averaged and minimized, even though it impinges on the correction capability. In the model the closed orbit is defined by the fitted kick angle (or momentum offset), of which the error is approximately $1 / \sqrt{N}$, with $N$ being the number of BPMs. This error can be minimized by repetition. However, the amplitude of the orbit offset is affected by the global gain factor of the BPMs, and the error cannot be reduced by repetition. During the analysis the BPM gain factors obtained from LOCO were applied.

While analyzing the simulated and the experimental data it was found that including the oscillation amplitude in the penalty function does not improve the precision. This is due to the poor accuracy of the amplitude determination.

\section{CONCLUSION}

In this paper we showed that beta function and nonlinear lattice can be corrected via betatron phase. Simulation results were presented to show the equivalence between the phase correction and the beta beat correction. An improvement was made in the Fourier transform analysis and the measurement accuracy of the phase was shown to be $1 \mathrm{mrad}$ (rms) at NSLS-II which corresponds to about $0.3 \%$ of rms beta beat. The beta beat of the NSLS-II storage ring can be corrected to approximately $1 \%$ in both planes and the results were confirmed by the LOCO measurement. The phase alone cannot be used to correct dispersion and coupling, however, this fast method is a good complement to the other lattice correction methods.

A scheme of nonlinear correction was proposed in the paper. The beam orbit can be offset in the sextupoles by an orbit corrector or by varying the rf frequency. By measuring the betatron phase change the sextupole strength error can be determined. It was shown in the paper that the phase change was related to all the leading order driving terms produced by sextupoles. By including the amplitude detuning terms and the chromatic detuning terms, all the necessary leading order terms are included for nonlinear optimization. It was successfully demonstrated at NSLS-II that a half percent sextupole correction led to a $15 \%$ increase of the dynamic aperture. The approach was further verified by experimentally identifying a known error.

\section{ACKNOWLEDGMENTS}

The authors are grateful to Eric Blum for editing the manuscript. The authors wish to thank Boris Podobedov, Weixing Cheng, and Steve Kramer for comments. Special thanks are due to the operations group for support on the machine studies. The work is supported by Brookhaven Science Associates, LLC under Contract No. DE-AC0298CH10886 with the U.S. Department of Energy.

\section{APPENDIX A: ERROR ANALYSIS OF THE FOURIER TRANSFORM}

In this section we study the error in the Fourier transformation in the presence of a frequency offset. Assume the signal is given by

$$
x_{n}=A \cos [(\nu+\Delta \nu) 2 n \pi+\psi]
$$

where $n=1,2, \ldots, N, \nu+\Delta \nu$ is the actual tune, and $\psi$ is the initial phase. Apply a Fourier transform at frequency $\nu$,

$$
\begin{aligned}
& a=\frac{2}{N} \sum_{n=1}^{N} x_{n} \cos (\nu 2 n \pi) \\
& b=\frac{2}{N} \sum_{n=1}^{N} x_{n} \sin (\nu 2 n \pi),
\end{aligned}
$$

where $\nu=k / N$ is a regular harmonic with $\mathrm{k}$ as an integer.

Using the following identities,

$$
\begin{gathered}
\sum_{n=1}^{N} \cos (n \theta+\psi)=\frac{\sin N \theta / 2}{\sin \theta / 2} \cos (N \theta / 2+\theta / 2+\psi) \\
\sum_{n=1}^{N} \sin (n \theta+\psi)=\frac{\sin N \theta / 2}{\sin \theta / 2} \sin (N \theta / 2+\theta / 2+\psi)
\end{gathered}
$$

we found 


$$
\begin{aligned}
a= & \frac{A \sin (N \Delta \nu \pi)}{N \sin (2 \nu+\Delta \nu) \pi} \cos \left[\psi_{N}+2 \nu \pi+\psi\right] \\
& +\frac{A \sin (N \Delta \nu \pi)}{N \sin \Delta \nu \pi} \cos \left[\psi_{N}+\psi\right] \\
b= & \frac{A \sin (N \Delta \nu \pi)}{N \sin (2 \nu+\Delta \nu) \pi} \sin \left[\psi_{N}+2 \nu \pi+\psi\right] \\
& -\frac{A \sin (N \Delta \nu \pi)}{N \sin \Delta \nu \pi} \sin \left[\psi_{N}+\psi\right]
\end{aligned}
$$

where $\psi_{N}=(N+1) \Delta \nu \pi$. Since $\Delta \nu \ll 1, a$ and $b$ are dominated by the second term. Keeping the second term only

$$
\begin{gathered}
a \approx \frac{A \sin (N \Delta \nu \pi)}{N \sin \Delta \nu \pi} \cos [(N+1) \Delta \nu \pi+\psi] \\
b \approx-\frac{A \sin (N \Delta \nu \pi)}{N \sin \Delta \nu \pi} \sin [(N+1) \Delta \nu \pi+\psi] .
\end{gathered}
$$

Therefore due to the frequency deviation the amplitude is scaled by a factor of $\frac{\sin (N \Delta \nu \pi)}{N \sin (\Delta \nu) \pi}$, and the phase changes by $(N+1) \Delta \nu \pi$. For the Fourier transform the maximum frequency error is $1 /(2 N)$, which leads to a scale factor of $2 / \pi$ and a phase error of $\pi / 2$. In other words, the Fourier transform is very inaccurate in determining the amplitude and phase. Fortunately in our application Eq. (6) defines the relative phase. Therefore the $(N+1) \Delta \nu \pi$ term is canceled between two BPMs. However, reducing $\Delta \nu$ still helps because it reduces the first term in Eqs. (A6) and (A7). The ratio of the first to the second term can be estimated from the function

$f^{2}(\nu, \Delta \nu, \psi) \approx \frac{\sin ^{2} \Delta \nu \pi}{\sin ^{2}(2 \nu+\Delta \nu) \pi} \approx 2(\Delta \nu \pi)^{2} \approx \frac{1}{2} \frac{\pi^{2}}{N^{2}}$

This term shows up in Eq. (11) as a random error contribution to the amplitude, and it leads to a phase error of about 1 milliradians if $\mathrm{N} \sim 1000$.

\section{APPENDIX B: THE DEPENDENCE OF PHASE ON THE DRIVING TERMS}

The beta beat introduced by quadrupolar errors can be approximated as

$$
\frac{\Delta \beta(s)}{\beta(s)}=-\frac{1}{2 \sin (2 \pi \nu)} \sum_{n} \Delta K_{1, n} \beta_{n} l_{n} \cos 2\left(\pi \nu-\left|\psi(s)-\psi_{n}\right|\right)
$$

The approximation is valid if $\Delta K_{1, n} l_{n}$ is small.
From the definition the phase advance $\psi(s)=\int \frac{d s}{\beta(s)}$, hence

$$
\begin{aligned}
\Delta \psi(s) & =\int d s\left(\frac{1}{\beta+\Delta \beta}-\frac{1}{\beta}\right) \\
& \approx-\int \frac{\Delta \beta(s)}{\beta^{2}(s)} d s=-\int \frac{\Delta \beta(s)}{\beta(s)} d \psi
\end{aligned}
$$

Define two step functions as follows

$$
\mathcal{Q}_{1}(x)= \begin{cases}1 & \text { if } x \geq 0 \\ 0 & \text { if } x<0\end{cases}
$$

and

$$
\mathcal{Q}_{2}(x)= \begin{cases}0 & \text { if } x \geq 0 \\ 1 & \text { if } x<0\end{cases}
$$

Apparently $\mathcal{Q}_{1}(x)+\mathcal{Q}_{2}(x)=1$. Then

$$
\begin{aligned}
\Delta \psi(s)= & \frac{1}{4 \sin (2 \pi \nu)} \sum_{n} \Delta K_{1, n} \beta_{n} l_{n} \\
& \times\left[\sin 2\left(\pi \nu-\psi_{n}+\psi(s)\right) \mathcal{Q}_{1}\left(\psi_{n}-\psi(s)\right)\right. \\
& -\sin 2\left(\pi \nu-\psi(s)+\psi_{n}\right) \mathcal{Q}_{2}\left(\psi_{n}-\psi(s)\right) \\
& \left.+2 \sin 2 \pi \nu-\sin \left(2 \pi \nu-2 \psi_{n}\right)\right] .
\end{aligned}
$$

The last two terms are integration constants and are determined from the tune change induced by the quadrupolar errors, i.e., $\Delta \nu=1 /(4 \pi) \sum_{n} \Delta K_{1, n} \beta_{n} l_{n}$.

The horizontal orbit distortion generated by a bending angle $\theta$ at $\psi_{x, 0}$ is given by

$$
\Delta x(s)=\frac{\sqrt{\beta_{x}(s) \beta_{x, 0}} \theta}{2 \sin \left(\pi \nu_{x}\right)} \cos \left(\pi \nu_{x}-\left|\psi_{x}(s)-\psi_{x, 0}\right|\right) .
$$

In order to simplify the notation, we choose $\psi_{x, 0}=0$. The focusing strength of a sextupole located at $\mathrm{s}$ is $\Delta K_{1}=K_{2} \Delta x(s)$, hence the phase change 


$$
\begin{aligned}
& \Delta \psi_{x}(s)=\sum_{n} \frac{\beta_{x, n}^{3 / 2} \beta_{x, 0}^{1 / 2}}{8 \sin \left(2 \pi \nu_{x}\right) \sin \left(\pi \nu_{x}\right)} K_{2, n} l_{n} \theta \\
& \times\left[\sin 2\left(\pi \nu_{x}+\psi_{x}(s)-\psi_{x, n}\right) \mathcal{Q}_{1}\left(\psi_{x, n}-\psi_{x}(s)\right)-\sin 2\left(\pi \nu_{x}+\psi_{x, n}-\psi_{x}(s)\right) \mathcal{Q}_{2}\left(\psi_{x_{n}}-\psi_{x}(s)\right)\right. \\
& \left.+2 \sin 2 \pi \nu_{x}-\sin \left(2 \pi \nu_{x}-2 \psi_{x, n}\right)\right] \cos \left(\pi \nu_{x}-\psi_{x, n}\right) \text {. } \\
& =\sum_{n} \frac{\beta_{x, n}^{3 / 2} \beta_{x, 0}^{1 / 2}}{8 \sin \left(2 \pi \nu_{x}\right) \sin \left(\pi \nu_{x}\right)} K_{2, n} l_{n} \theta\left\{\left[\sin \left(3 \pi \nu_{x}-3 \psi_{x, n}+2 \psi_{x}(s)\right)+\sin \left(\pi \nu_{x}-\psi_{x, n}+2 \psi_{x}(s)\right)\right] \mathcal{Q}_{1}\left(\psi_{x, n}-\psi_{x}(s)\right)\right. \\
& -\left[\sin \left(3 \pi \nu_{x}+\psi_{x, n}-2 \psi_{x}(s)\right)+\sin \left(\pi \nu_{x}-2 \psi_{x}(s)+3 \psi_{x, n}\right)\right] \mathcal{Q}_{2}\left(\psi_{x, n}-\psi_{x}(s)\right) \\
& \left.+2 \sin \left(3 \pi \nu_{x}-\psi_{x, n}\right)+2 \sin \left(\pi \nu_{x}+\psi_{x, n}\right)-\sin \left(3 \pi \nu_{x}-3 \psi_{x, n}\right)-\sin \left(\pi \nu_{x}-\psi_{x, n}\right)\right\} \\
& =\sum_{n} \frac{\beta_{x, n}^{3 / 2} \beta_{x, 0}^{1 / 2}}{8 \sin \left(2 \pi \nu_{x}\right) \sin \left(\pi \nu_{x}\right)} K_{2, n} l_{n} \theta \operatorname{Im}\left\{\left[e^{i\left(3 \pi \nu_{x}+2 \psi_{x}(s)\right)-3 i \psi_{x, n}}+e^{i\left(\pi \nu_{x}+2 \psi_{x}(s)\right)-i \psi_{x, n}}\right] \mathcal{Q}_{1}\left(\psi_{x, n}-\psi_{x}(s)\right)\right. \\
& -\left[e^{i\left(3 \pi \nu_{x}-2 \psi_{x}(s)\right)+i \psi_{x, n}}+e^{i\left(\pi \nu_{x}-2 \psi_{x}(s)\right)+3 i \psi_{x, n}}\right] \mathcal{Q}_{2}\left(\psi_{x, n}-\psi_{x}(s)\right) \\
& \left.+2 e^{3 i \pi \nu_{x}-i \psi_{x, n}}+2 e^{i \pi \nu_{x}+i \psi_{x, n}}-e^{3 i \pi \nu_{x}-3 i \psi_{x, n}}-e^{i \pi \nu_{x}-i \psi_{x, n}}\right\} \\
& =\frac{\beta_{x, 0}^{1 / 2} \theta}{8 \sin \left(2 \pi \nu_{x}\right) \sin \left(\pi \nu_{x}\right)} \operatorname{Im}\left\{\left[e^{i\left(3 \pi \nu_{x}+2 \psi_{x}(s)\right)} h_{03000}+e^{i\left(\pi \nu_{x}+2 \psi_{x}(s)\right)} h_{12000}\right] \mathcal{Q}_{1}\left(\psi_{x, n}-\psi_{x}(s)\right)\right. \\
& -\left[e^{i\left(3 \pi \nu_{x}-2 \psi_{x}(s)\right)} h_{21000}+e^{i\left(\pi \nu_{x}-2 \psi_{x}(s)\right)} h_{30000}\right] \mathcal{Q}_{2}\left(\psi_{x, n}-\psi_{x}(s)\right) \\
& \left.+\left(2 e^{3 i \pi \nu_{x}}-e^{i \pi \nu_{x}}\right) h_{12000}+2 e^{i \pi \nu_{x}} h_{21000}-e^{3 i \pi \nu_{x}} h_{03000}\right\} \text {. }
\end{aligned}
$$

Similarly the vertical phase change due to the same horizontal orbit distortion reads

$$
\begin{aligned}
\Delta \psi_{y}(s)= & \frac{\beta_{x, 0}^{1 / 2} \theta}{8 \sin \left(2 \pi \nu_{y}\right) \sin \left(\pi \nu_{x}\right)} \operatorname{Im}\left\{\left[e^{i\left(2 \pi \nu_{y}+\pi \nu_{x}+2 \psi_{y}(s)\right)} h_{01020}+e^{i\left(2 \pi \nu_{y}-\pi \nu_{x}+2 \psi_{y}(s)\right)} h_{10020}\right] \mathcal{Q}_{1}\left(\psi_{x, n}-\psi_{x}(s)\right)\right. \\
& -\left[e^{i\left(2 \pi \nu_{y}+\pi \nu_{x}-2 \psi_{y}(s)\right)} h_{01200}+e^{i\left(2 \pi \nu_{y}-\pi \nu_{x}-2 \psi \psi_{y}(s)\right)} h_{10200}\right] \mathcal{Q}_{2}\left(\psi_{x, n}-\psi_{x}(s)\right) \\
& \left.+2\left[e^{i\left(2 \pi \nu_{y}+\pi \nu_{x}\right)} h_{01110}+e^{i\left(2 \pi \nu_{y}-\pi \nu_{x}\right)} h_{10110}\right]-\left[e^{i\left(2 \pi \nu_{y}+\pi \nu_{x}\right)} h_{01020}+e^{i\left(2 \pi \nu_{y}-\pi \nu_{x}\right)} h_{10020}\right]\right\} .
\end{aligned}
$$

Here the geometric driving terms are defined as [31]

$$
\begin{aligned}
& h_{21000}=h_{12000}^{*}=\sum_{n} K_{2, n} l_{n} \beta_{x, n}^{3 / 2} e^{i \psi_{x, n}} \\
& h_{30000}=h_{03000}^{*}=\sum_{n} K_{2, n} l_{n} \beta_{x, n}^{3 / 2} e^{i 3 \psi_{x, n}} \\
& h_{10110}=h_{01110}^{*}=\sum_{n} K_{2, n} l_{n} \beta_{x, n}^{1 / 2} \beta_{y, n} e^{i \psi_{x, n}} \\
& h_{10020}=h_{01200}^{*}=\sum_{n} K_{2, n} l_{n} \beta_{x, n}^{1 / 2} \beta_{y, n} e^{i\left(\psi_{x, n}-2 \psi_{y, n}\right)} \\
& h_{10200}=h_{01020}^{*}=\sum_{n} K_{2, n} l_{n} \beta_{x, n}^{1 / 2} \beta_{y, n} e^{i\left(\psi_{x, n}+2 \psi_{y, n}\right)} .
\end{aligned}
$$

Now consider the phase change caused by a momentum offset $\delta=\Delta p / p$. The focusing strength becomes

$$
\Delta K_{1}=K_{2} D_{1}(s) \delta
$$

Here only the first order dispersion $D_{1}(s)$ is taken into account. The phase changes are 


$$
\begin{aligned}
\Delta \psi_{x}(s)= & \sum_{n} \frac{\beta_{x, n}}{4 \sin \left(2 \pi \nu_{x}\right)} K_{2, n} l_{n} \theta\left\{\sin 2\left[\pi \nu_{x}+\psi_{x}(s)-\psi_{x, n}\right] \mathcal{Q}_{1}\left[\psi_{x, n}-\psi_{x}(s)\right]-\sin 2\left[\pi \nu_{x}+\psi_{x, n}-\psi_{x}(s)\right] \mathcal{Q}_{2}\left[\psi_{x_{n}}-\psi_{x}(s)\right]\right. \\
& \left.+2 \sin 2 \pi \nu_{x}-\sin \left(2 \pi \nu_{x}-2 \psi_{x, n}\right)\right\} D_{1, n} \delta . \\
= & \frac{\theta}{4 \sin \left(2 \pi \nu_{x}\right)} \operatorname{Im}\left\{e^{i\left(2 \pi \nu_{x}+2 \psi_{x}(s)\right)} h_{02001} \mathcal{Q}_{1}\left[\psi_{x, n}-\psi_{x}(s)\right]+e^{i\left(2 \pi \nu_{x}-2 \psi_{x}(s)\right)} h_{20001} \mathcal{Q}_{2}\left[\psi_{x, n}-\psi_{x}(s)\right]\right. \\
& \left.+2 e^{i 2 \pi \nu_{x}} h_{11001}-e^{i 2 \pi \nu_{x}} h_{02001}\right\} \\
\Delta \psi_{y}(s)= & \sum_{n} \frac{\beta_{y, n}}{4 \sin \left(2 \pi \nu_{y}\right)} K_{2, n} l_{n} \theta\left\{\sin 2\left[\pi \nu_{y}+\psi_{y}(s)-\psi_{y, n}\right] \mathcal{Q}_{1}\left[\psi_{y, n}-\psi_{y}(s)\right]-\sin 2\left[\pi \nu_{y}+\psi_{y, n}-\psi_{y}(s)\right] \mathcal{Q}_{2}\left[\psi_{y_{n}}-\psi_{y}(s)\right]\right. \\
& \left.+2 \sin 2 \pi \nu_{y}-\sin \left(2 \pi \nu_{y}-2 \psi_{y, n}\right)\right\} D_{1, n} \delta . \\
= & \frac{\theta}{4 \sin \left(2 \pi \nu_{x}\right)} \operatorname{Im}\left\{e^{i\left(2 \pi \nu_{y}+2 \psi_{y}(s)\right)} h_{00021} \mathcal{Q}_{1}\left[\psi_{y, n}-\psi_{y}(s)\right]+e^{i\left[2 \pi \nu_{y}-2 \psi_{y}(s)\right]} h_{00201} \mathcal{Q}_{2}\left[\psi_{y, n}-\psi_{y}(s)\right]\right. \\
& \left.+2 e^{i 2 \pi \nu_{y}} h_{00111}-e^{i 2 \pi \nu_{y}} h_{00021}\right\} .
\end{aligned}
$$

The chromatic driving terms are defined as

$$
\begin{aligned}
& h_{11001}=\sum_{n} K_{2, n} l_{n} \beta_{x, n} D_{1, n} \\
& h_{00111}=\sum_{n} K_{2, n} l_{n} \beta_{y, n} D_{1, n} \\
& h_{20001}=h_{02001}^{*}=\sum_{n} K_{2, n} l_{n} \beta_{x, n} D_{1, n} e^{i 2 \psi_{x, n}} \\
& h_{00201}=h_{00021}^{*}=\sum_{n} K_{2, n} l_{n} \beta_{y, n} D_{1, n} e^{i 2 \psi_{y, n}} .
\end{aligned}
$$

We would like to point out that (1) In the analysis the corrector is treated as the reference point. If the reference point is fixed an additional phase term shows up in all the driving terms. This phase term varies from 0 to $2 \pi$ when the correctors are switched. Consequently the phase depends on the imaginary part and the real part of the driving terms even though in the formulas only the imaginary part is shown. (2) $\Delta \psi_{x}, \Delta \psi_{y}$ cover all but one of the leading order driving terms provided the on- and off-momentum phases are considered. The only missing term is $h_{10002}$, which is a $\delta^{2}$ term and can be regarded as higher order. (3) The terms that are multiplied by $\mathcal{Q}_{1,2}\left[\psi_{n}-\psi(s)\right]$ mean the summation is between the corrector and the observation point $\psi(s)$. Be aware that the driving terms are defined for a full turn. Therefore the driving terms here are sectional driving terms. However, if the driving terms are restored in every section, the sum for the whole ring must be corrected. Consequently these terms can be perceived as regular driving terms for our purpose. (4) On the other hand, due to these $\mathcal{Q}_{1,2}\left[\psi_{n}-\psi(s)\right]$ terms the coefficients of these driving terms are linearly independent when switching correctors. Therefore correcting the phase advance will lead to restoring the driving terms. This convergence has been verified in simulation.

[1] M. Bai, S. Y. Lee, J. W. Glenn, H. Huang, L. Ratner, T. Roser, M. J. Syphers, and W. van Asselt, Experimental test of coherent betatron resonance excitations, Phys. Rev. E 56, 6002 (1997).

[2] D. Sagan, Betatron phase and coupling correction at the Cornell Electron/Positron Storage Ring, Phys. Rev. ST Accel. Beams 3, 102801 (2000).

[3] R. Tomas, Adiabaticity of the ramping process of an ac dipole, Phys. Rev. ST Accel. Beams 8, 024401 (2005).

[4] R. Miyamoto, A. Jansson, M. J. Syphers, and S. E. Kopp, Nonlinear dynamics studies in the Fermilab Tevatron using AC dipole, in Proceedings of the 23rd Particle Accelerator Conference, Vancouver, Canada, 2009 (IEEE, Piscataway, NJ, 2009), p. 3071, http://accelconf.web.cern.ch/ AccelConf/PAC2009/papers/th2pbc02.pdf.

[5] X. Yang, V. Smaluk, L. H. Yu, Y. Tian, and K. Ha, Fast and precise technique for magnet lattice correction via sinewave excitation of fast correctors, Phys. Rev. Accel. Beams 20, 054001 (2017). 
[6] I. P. S. Martin, M. Abbott, M. Furseman, G. Rehm, and R. Bartolini, A fast optics correction for the Diamond storage ring, in Proceedings of IPAC'14, Dresden, Germany, p. 1763, http://accelconf.web.cern.ch/AccelConf/ IPAC2014/papers/tupri083.pdf.

[7] J. Safranek, Experimental determination of storage ring optics using orbit response measurements, Nucl. Instrum. Methods Phys. Res., Sect. A 388, 27 (1997); M. Harrison and S. Peggs, Global beta measurement from two perturbed closed orbits, in Proceedings of the 1987 Particle Accelerator Conference (IEEE, Washington, DC, 1987), p. 1105; Y. Chung, G. Decker, and K. Evans, Measurement of betafunction and phase using the response matrix, in Proceedings of the 15th Particle Accelerator Conference, PAC1993, Washington, DC, 1993 (IEEE, New York, 1993), p. 118; W. J. Corbett, M. J. Lee, and V. Ziemann, A fast model-calibration procedure for storage rings, in Proceedings of the 15th Particle Accelerator Conference, PAC1993, Washington, DC (IEEE, New York, 1993), p. 108.

[8] Beam Dynamics Newsletter 44, International Committee for Future Accelerators (ICFA), (2007).

[9] P. Castro, J. Borer, A. Burns, G. Morpurgo, and R. Schmidt, Betatron function measurement at LEP using the BOM 1000 turns facility, in Proceedings of the 15th Particle Accelerator Conference, PAC-1993, Washington, DC, 1993 (IEEE, New York, 1993), p. 2103, http://accelconf.web.cern.ch/ AccelConf/p93/PDF/PAC1993_2103.PDF.

[10] R. Tomas, O. Bruning, S. Fartoukh, M. Giovannozzi, Y. Papaphilippou, F. Zimmermann, R. Calaga S. Peggs, and A. Franchi, Procedures and accuracy estimates for betabeat correction in the LHC, in Proceedings of the 10th European Particle Accelerator Conference, Edinburgh, Scotland, 2006 (EPS-AG, Edinburgh, Scotland, 2006), p. 2023, http://accelconf.web.cern.ch/AccelConf/e06/ PAPERS/WEPCH047.PDF.

[11] X. Huang, Application of independent component analysis to Fermilab Booster, Phys. Rev. ST Accel. Beams 8, 064001 (2005).

[12] G. Wang, M. Bai, V. N. Litvinenko, and T. Satogata, The correction of linear lattice gradient errors using an AC dipole, in Proceedings of the 23rd Particle Accelerator Conference, Vancouver, Canada, 2009 (IEEE, Piscataway, NJ, 2009), p. 3859, http://accelconf.web.cern.ch/ AccelConf/PAC2009/papers/th6pfp066.pdf.

[13] G. Rehm, M. G. Abbott, A. F. D. Morgan, J. Rowland, and I. Uzun, Measurement of lattice parameters without visible disturbance to user beam at Diamond light source, in Proceedings of BIW10, Santa Fe, New Mexico, 2010, pp. 44-48, http://accelconf.web.cern.ch/AccelConf/ BIW2010/papers/mocnb01.pdf.

[14] M. Aiba, M. Böge, J. Chrin, N. Milas, T. Schilcher, and A. Streun, Comparison of linear optics measurement and correction methods at the Swiss Light Source, Phys. Rev. ST Accel. Beams 16, 012802 (2013).

[15] L. Malina, J. Coello de Portugal, T. Persson, P. K. Skowroński, R. Tomás, A. Franchi, and S. Liuzzo, Improving the precision of linear optics measurements based on turn-by-turn beam position monitor data after a pulsed excitation in lepton storage rings, Phys. Rev. Accel. Beams 20, 082802 (2017).
[16] R. Tomás, M. Aiba, A. Franchi, and U. Iriso, Review of linear optics measurement and correction for charged particle accelerators, Phys. Rev. Accel. Beams 20, 054801 (2017).

[17] G. M. Wang, T. Shaftan, F. Willeke et al., in NSLS II Commissioning report, BNL Technical Note 168, 2015, p. 37.

[18] Y. Li, L. Yang, and W. Cheng, Proceedings of ICAP2015, Shanghai, China, 2015, pp. 6-8, http://accelconf.web.cern .ch/AccelConf/ICAP2015/papers/modbc1.pdf.

[19] A. Franchi, L. Farvacque, F. Ewald, G. Le Bec, and K. B. Scheidt, First simultaneous measurement of sextupolar and octupolar resonance driving terms in a circular accelerator from turn-by-turn beam position monitor data, Phys. Rev. ST Accel. Beams, 17, 074001 (2014).

[20] R. Bartolini, I. P. S. Martin, J. H. Rowland, P. Kuske, and F. Schmidt, Correction of multiple nonlinear resonances in storage rings, Phys. Rev. ST Accel. Beams 11, 104002 (2008).

[21] J. Bengtsson, I. P. S. Martin, J. H. Rowland, and R. Bartolini, On-line control of the nonlinear dynamics for synchrotrons, Phys. Rev. ST Accel. Beams 18, 074002 (2015).

[22] R. Bartolini, I. P. S. Martin, G. Rehm, and F. Schmidt, Calibration of the nonlinear ring model at the Diamond Light Source, Phys. Rev. ST Accel. Beams 14, 054003 (2011).

[23] J. Feikes, J. Kuszynski, and G. Wüstefeld, Beam lifetime optimization of BessyII by systematically adjusting the harmonic sextupoles, in Proceedings of the 8th European Particle Accelerator Conference, Paris, 2002 (EPS-IGA and CERN, Geneva, 2002), p. 775, http://accelconf.web .cern.ch/AccelConf/e02/PAPERS/TUPRI024.pdf.

[24] X. Huang and J. Safranek, Online optimization of storage ring nonlinear beam dynamics, Phys. Rev. ST Accel. Beams 18, 084001 (2015).

[25] W. Guo, B. Yang, C.-x. Wang, K. Harkay, and M. Borland, Generating picosecond $\mathrm{X}$-ray pulses in synchrotron light sources using dipole kickers, Phys. Rev. ST Accel. Beams 10, 020701 (2007).

[26] Y. Shoji, M. Takao, and T. Nakamura, Amplitude dependent shift of betatron oscillation center, Phys. Rev. ST Accel. Beams 17, 064001 (2014).

[27] M. Borland, elegant: A flexible SDDS-compliant code for accelerator simulation, Advanced Photon Source Report No. LS-287, 2000.

[28] J. Laskar, C. Froeschle, and A. Celletti, The measure of chaos by the numerical analysis of the fundamental frequencies. Application to the standard mapping, Physica D (Amsterdam) 56, 253 (1992).

[29] W. X. Cheng et al., Characterization of NSLS2 storage ring beam orbit stability, in Proceedings of IBIC2015, Melbourne, Australia, THALA02, p. 625, http://accelconf.web .cern.ch/AccelConf/IBIC2015/papers/thala02.pdf.

[30] V. Smalyuk, X. Yang, W. Guo, Y. Hidaka, G. Wang, Y. Li, and L. Yang, Experimental crosscheck of algorithms for magnet lattice correction, in Proceedings of IPAC2016, Busan, Korea, p. 3400, http://accelconf.web.cern.ch/ AccelConf/ipac2016/papers/thpmr008.pdf.

[31] J. Bengtsson, The sextupole scheme for the Swiss Light Source (SLS): An Analytic Approach, SLS Note 9/97, 1997 (unpublished). 